\title{
URBAN TRAFFIC SIMULATION WITH PSYCHO-PHYSICAL VEHICLE-FOLLOWING MODELS
}

\author{
Thomas Schulze \\ Technical University Dresden \\ Faculty for Computer Sciences \\ Department for Information Systems \\ D-01307 Dresden, GERMANY
}

\author{
Thomas Fliess \\ Otto-Guericke-University Magdeburg \\ Faculty for Computer Sciences \\ Department of Simulation and Graphics \\ D-39106 Magdeburg, PSF 4120, GERMANY
}

\section{ABSTRACT}

A psycho-physical vehicle-following model tries to capture both the physical and human components of congested-traffic simulations. These models determine the reactions of the vehicle driver depending on the vehicle's state. The state of a vehicle can be determined by the distance and by the difference in speed, in comparison to the leading vehicle. If the vehicle state changes, the driver must react. The reaction of the driver is to calculate a new value for acceleration. There are two different classical methods of time advance to calculate the state changes. This paper examines the effect of these methods on simulation run times and simulation results. Empirical experiments were done on a SLX-based simulation model.

\section{INTRODUCTION}

Traffic simulation has been a wide application area for modeling and simulation for many years. Both analytical and simulation models are used depending on the aims of traffic modeling. Simulation models have been frequently applied in the area of urban street traffic. Existing modeling approaches can be classified depending on the level of detail for the simulated process. Macroscopic approaches describe the traffic flow in the entirety of all vehicles (Kuehne 1995). Significant parameters are traffic density, flow of traffic and so on. The main application area for this kind of approach is the simulation of wide-area traffic systems like highway networks and interregional road networks.

Microscopic approaches are characterized by the description of single vehicles and relations between them (Lieberman 1991). Models from this class show the behavior of single traffic participants. Valid traffic rules and regulations form the basis for the applied behavior rules. The traffic rules define, for example, the maximum of speed and the right of way. The behavior rules contain additional strategies for controlling speed and acceleration. Currently, microscopic models are used to simulate urban traffic processes on single intersections or on networks of urban intersections.

For many years analytical models have been used in the field of microscopic traffic modeling. The application of analytical models is, however, subject to certain conditions such as homogeneity of the arrival process. Rough estimates of the performance parameters can be done with this model class.

Intelligent load-dependent control approaches for traffic lights are used by traffic engineers to manage the increasing urban volume of traffic (Hoyer 1993). Simulation models are necessary tools for evaluating the quality of load-dependent controls in project phases. Traffic engineers demand from the simulation side:

- simulation models with a high level of detail,

- fast simulation models, and

- acceptable expenses for the model building process.

The needed level of detail is focused on modelling the vehicle-following process and rules for resolving conflicts between vehicles, like who has the right of way. Queuing models or psycho-physical modelling approaches are widely used for modelling vehiclefollowing processes.

Queuing models (Schlothauer, Schulze 1996) describe parts of a road as single- or multiple-server systems with queue capacity of zero. Arriving entities (vehicles) can be served only if at least one necessary server is idle. The service time for a new entity depends on the speed of the preceding vehicle. Passing the predecessors is not allowed. The simple structure of queuing models does not permit modelling the vehicle-acceleration process. The speed of a vehicle is used as a discrete parameter in the model. The use of psycho-physical vehicle-following models leads to a higher level of model accuracy. 


\section{PSYCHO-PHYSICAL VEHICLE FOLLOWING MODELS}

Psycho-physical vehicle-following models determine reactions of vehicles in traffic processes with regard to changing the acceleration of vehicles. Reactions happen if differences in speed and distance to the predecessor occur (Wiedemann 1974). Different simulators have been developed and implemented, based on the ideas of Wiedemann. A new SLX-based (Heriksen 1996) psychophysical vehicle-following simulation model has been developed and integrated within a new microscopic traffic simulator. Development has been done at the University of Magdeburg (Fliess 1996).

Vehicles are the main objects in this modeling approach. The class of vehicles contains the following default attributes:

- current position on a path,

- current speed,

- current acceleration,

- desired speed,

- maximum negative acceleration,

- maximum positive acceleration and

- current state.

There are only discrete values for the current state attribute. The state of a vehicle describes six different behavioral patterns of vehicles. The state will be changed if defined limits for distances to the predecessor or differences in speed are crossed. The acceleration of the vehicle will be newly recalculated and shall then be constant until the driver has to react to new traffic conditions. Table 1 lists the different limits for the distance to the predecessor (leading vehicle). Limits for distances depend on the current speed of the vehicle and a vehicle specific time gap.

Table 1: Limits for the Distance to the Leading Vehicle

\begin{tabular}{|l|l|c|}
\hline Limits & Independent Variables & $\begin{array}{c}\text { Values for Time } \\
\text { Gaps [sec] }\end{array}$ \\
\hline $\begin{array}{l}\text { break } \\
\text { distance }\end{array}$ & $\begin{array}{l}\text { own speed, } \\
\text { time gap to break }\end{array}$ & 2.5 \\
\hline $\begin{array}{l}\text { desired } \\
\text { distance }\end{array}$ & $\begin{array}{l}\text { own speed, } \\
\text { desired time gap }\end{array}$ & 1.8 \\
\hline $\begin{array}{l}\text { safety } \\
\text { distance }\end{array}$ & $\begin{array}{l}\text { own speed } \\
\text { time gap for safety }\end{array}$ & 0.5 \\
\hline $\begin{array}{l}\text { risk } \\
\text { distance }\end{array}$ & $\begin{array}{l}\text { speed of the prede-cessor, } \\
\text { time gap for distance }\end{array}$ & 1.0 \\
\hline
\end{tabular}

For example, the limit for the desired distance can be calculated with

$$
\Delta x_{d d}=T_{d} * v_{1}+D_{0}
$$

where $\Delta x_{d d}$ denotes the limit for the desired distance, $T_{d}$ denotes the individual desired time gap. $v$, denotes the current speed of vehicle $i$ and $D_{0}$ denotes the distance if two vehicles are standing one after the other.

Limits for differences in speed to the leading vehicle can be classified into two cases. These cases are described in Table 2.

Table 2: Limits for the Differences in Speed

\begin{tabular}{|l|l|}
\hline Limit & Independent Variables \\
\hline $\begin{array}{l}\text { positive observation limit } \\
\Delta v>0\end{array}$ & $\begin{array}{l}\text { distance to the leading } \\
\text { vehicle }\end{array}$ \\
\hline $\begin{array}{l}\text { negative observation limit } \\
\Delta v<0\end{array}$ & $\begin{array}{l}\text { distance to the leading } \\
\text { vehicle }\end{array}$ \\
\hline
\end{tabular}

The positive observation limit can be calculated with

$$
\Delta_{\text {lpos }}=k_{1}\left(\Delta x-k_{3}\right)^{2}+k_{4}
$$

where $\Delta x$ denotes the distance to the leading vehicle and $k_{1}, k_{2}, k_{3}$ denote special constants.

The different limits used can be shown in an observation-decision diagram. Difference in speed scales the abscissa and difference in distance scales the ordinate of the diagram. Such a diagram shows the possible states and crossings over the states for vehicles. Figure 1 presents an observation-decision diagram with possible values. The vehicle states used are presented in Table 3.

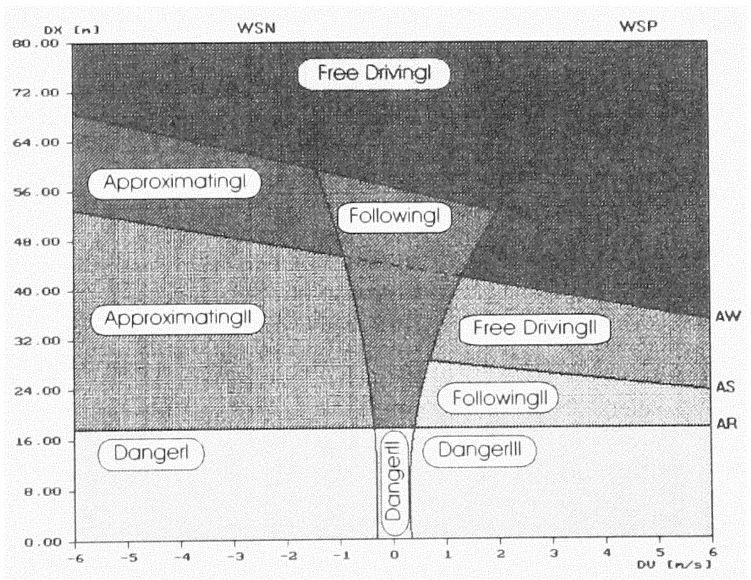

Fig. 1: Observation-Decision Diagram

A new value for the acceleration attribute must be calculated if the vehicle has changed its state. For 
example, the acceleration in the state Approximatingl will be calculated by

$$
a_{i}=\frac{\left(\Delta x_{1}-D_{0}-T_{d} v_{1}\right) a_{1-1}-0.5 \Delta v_{1}^{2}}{\Delta x_{1}-D_{0}-T_{d} v_{1-1}}
$$

where $\Delta x$, denotes the distance between the leading and following vehicle, $v$, denotes the current speed of the following vehicle, and $a_{1-1}$ is the acceleration of the leading vehicle.

Table 3: Description of vehicle states

\begin{tabular}{|l|l|l|}
\hline State & Kind of drive & Acceleration \\
\hline $\begin{array}{l}\text { Free } \\
\text { DrivingI }\end{array}$ & $\begin{array}{l}\text { not } \\
\text { influenced }\end{array}$ & $\begin{array}{l}\text { depending on the current } \\
\text { speed and the desired } \\
\text { speed }\end{array}$ \\
\hline $\begin{array}{l}\text { Free } \\
\text { DrivingII }\end{array}$ & $\begin{array}{l}\text { direct } \\
\text { influenced }\end{array}$ & $\begin{array}{l}\text { positive acceleration } \\
\text { until the desired distance } \\
\text { is reached and the dif- } \\
\text { ference in speed is zero }\end{array}$ \\
\hline $\begin{array}{l}\text { Approxi- } \\
\text { matingI }\end{array}$ & $\begin{array}{l}\text { direct } \\
\text { influenced }\end{array}$ & $\begin{array}{l}\text { negative acceleration } \\
\text { until the desired distance } \\
\text { is reached and the dif- } \\
\text { ference in speed is zero }\end{array}$ \\
\hline $\begin{array}{l}\text { Approxi- } \\
\text { matingII }\end{array}$ & $\begin{array}{l}\text { direct } \\
\text { influenced } \\
\text { until the risk distance is } \\
\text { reached and the differ- } \\
\text { ence in speed is zero }\end{array}$ \\
\hline FollowingI & $\begin{array}{l}\text { indirect } \\
\text { influenced }\end{array}$ & $\begin{array}{l}\text { keeping acceleration } \\
\text { until the desired distance } \\
\text { is reached and the differ- } \\
\text { ence in speed is zero }\end{array}$ \\
\hline
\end{tabular}

Figure 2 shows the progress of the trajectory

$$
T=f(\text { distance }(t), \text { difference in speed }(t))
$$

for the following vehicle until the modeling time reaches $100 \mathrm{sec}$. The values used for the vehicle attributes are presented in Table 4 .

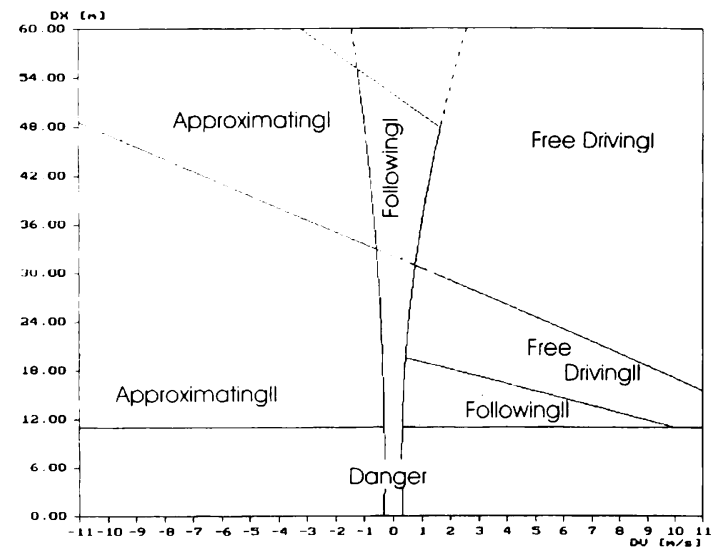

Fig. 2: Trajectory Progress for the Following Vehicle

\section{IMPLEMENTING TIME ADVANCES}

There are two methods of implementing time advances in simulation models. These methods are called the timesliced or the event-oriented methods. Using the timesliced-oriented approach, the attribute values of vehicles will be updated at constant time slices. At a constant rate the simulator checks for all vehicles if a limit has been crossed and the vehicle must get a new state. The selected value in existing traffic simulators for the time slice is often $1.0 \mathrm{sec}$. This value was selected according to the mean reaction time of a driver. The time-sliceoriented method can be implemented very easily.

Table 4: Selected Attributes for Vehicles at Time 0.0

\begin{tabular}{|l|l|c|}
\hline Vehicle & Attributes & Values \\
\hline Leading Vehicle & current position & $55.0 \mathrm{~m}$ \\
& current speed & $20.0 \mathrm{~m} / \mathrm{s}$ \\
& acceleration & $0.0 \mathrm{~m} / \mathrm{s}^{2}$ \\
\hline Following & current position & $0.0 \mathrm{~m}$ \\
Vehicle & current speed & $30.0 \mathrm{~m} / \mathrm{s}$ \\
& acceleration & $0.0 \mathrm{~m} / \mathrm{s}^{2}$ \\
\hline
\end{tabular}

Using the event-oriented method, the simulator has to compute the time when a vehicle will change its state. The time for this event can be calculated from the intersection of vehicle's trajectory with corresponding limits. The necessary condition is: There is no change in the state of the leading vehicle.

Determination of intersections between the trajectory and limits for the distance can be reduced to an intersection between a parabola and a line. To do this, the simulator has to solve a second-degree equation. The intersection between the trajectory and limits for differences in speed will be reduced to solving a fourthdegree equation. Suitable approximation methods like 
Newton iteration can be used for determining the event time. These events are called expected events. Table 5 presents a list of state events for the following vehicle using the attribute values of Table 4 .

Table 5: List of Events for the Following Vehicle

\begin{tabular}{|l|l|r|}
\hline Clock Value & Vehicle State & Acceleration $\left[\mathrm{m} / \mathrm{s}^{2}\right]$ \\
\hline 0.0 & ApproximatingI & -1.27 \\
\hline 3.2 & ApproximatingII & -1.27 \\
\hline 10.41 & Followingl & -1.27 \\
\hline 10.91 & FollowingII & -1.27 \\
\hline 13.63 & Free DrivingII & 0.46 \\
\hline 18.62 & Free DrivingII & 0.46 \\
\hline 20.09 & FollowingI & 0.46 \\
\hline 21.80 & FollowingI & 0.00 \\
\hline
\end{tabular}

Using the event oriented method, the calculation for new attribute values of involved vehicles has been done eight times. Ideal conditions lead to these small number of calculations: There are only two vehicles in the system and the state of the leading vehicle is constant. Using the time sliced approach with a time slice of one sec., the calculation of new attributes has been done 22 times for both vehicles.

Realistic traffic processes are characterized by a larger number of vehicles and frequent state changes of the leading vehicle. So the simulator has to process both expected and unexpected events. Unexpected events occur if the leading vehicle changes its state and the following vehicle has to react to the new values of attributes of the leading vehicle. A reaction of the following vehicle will take place after passing a reaction time.

State changes of the leading vehicle have several consequences. The simulator has to proceed with the following steps:

1. Calculation of the new time for the new expected event for the leading vehicle.

2. Scheduling this event into the event list.

3. Remove the expected event for the following vehicle from the event list.

4. Calculation of the unexpected event for following vehicle.

5. Scheduling this event into the event list.

Steps 1 and 4 contain the determination of the intersection between the trajectory of the leading vehicle with corresponding limits. The approximation of the intersection between the trajectory and the limits for differences in speed is a compute-intensive process.

Steps 2, 3 and 5 concern operations with the event list of the simulator. These actions involve basic operations for removing and inserting events. The run times for these operations depend on the number of events in the event list, the internal scheduling algorithms and the implementation level of these algorithms (Schulze and Preuß 1997).

The number of events will be determined by the number of moving vehicles in the traffic system. Event lists of urban traffic simulators contain not only events for vehicles. For example, they contain events for street cars, bicycles, pedestrians and traffic lights. An average content of more than 200 events is typical for urban traffic systems.

\section{REMARKS ON SIMULATION RUN TIMES}

Run times for simulation models are affects by different factors. On one hand, the use of constantly increasing processor power reduces the run time. On the other hand, the desired higher level of modeling detail leads to increasing run times for simulation experiments. A lot of runs have to be executed for constructing acceptably small confidential intervals. The input data for arriving vehicles are characterized by large dispersion. Our experience in typical traffic simulations is that the number of necessary runs is often greater than 20 . Results have to describe the steady-state of the traffic system. The influence of the transient phase should be rejected. Experiences from real traffic-simulation projects show that the length of the transient phase can be as much as 60 minutes of simulated time. This value was used for urban-traffic systems with three networked intersections. The minimum length of one simulation run was defined to be 120 simulated minutes. The need for a lot of simulation runs and the necessary simulation length require fast computer run time for executing simulation experiments.

The computer run time for traffic simulation models can be divided into two parts:

- Basic demand for static objects and

- Additional demand for dynamic objects.

The basic demand includes the time for initialization of the static model components like parameters of roads and lanes, or timing for traffic lights. The additional demand includes the time for:

- creating and destroying dynamic objects,

- resolving conflicts between different road users, like yielding and pedestrians getting in a car's way, and

- vehicle following. 
The run time for slice-oriented or event-oriented methods depends on different factors. The dependencies are shown in Table 6 .

Table 6: Dependencies on Run Time for Traffic Models

\begin{tabular}{|l|l|}
\hline $\begin{array}{c}\text { Method of Time } \\
\text { Advances }\end{array}$ & \multicolumn{2}{|c|}{ Dependencies on run time } \\
\hline slice oriented & $\bullet \begin{array}{l}\text { Number of dynamic model } \\
\text { objects } \\
\text { Slice value }\end{array}$ \\
\hline event oriented & $\bullet \begin{array}{l}\text { Number of dynamic model } \\
\text { objects }\end{array}$ \\
& $\bullet \begin{array}{l}\text { Number of events } \\
\text { Implementation level of event- } \\
\text { list-handling algorithms }\end{array}$ \\
\hline
\end{tabular}

The number of dynamic objects affects both methods of time advance. An increased number of dynamic objects leads to longer run time because more attribute values of these objects have to be updated. Reduction of the time slice value results in increased run time in the time-slice approach.

The computer time in the event-oriented approach is very strongly influenced by the algorithm used for eventlist handling and its implementation (McCormack 1979, Schulze and Preuß 1997). Computer-time efficientalgorithms are necessary for processing a large number of events.

The number of events to process depends on the number of dynamic modeling objects, as well as the interval of time between two successive events for a dynamic object. Short intervals between occurrence of events leads to a growth of both the number of state changes and event-list updates. The frequency of state changes depends on the functions describing the limits and on the operations during a state change.

The following hypothesis can be derived from this considerations above: If the average interval for a state change is greater than the value for the time slice, then the run time for the event oriented-approach is smaller than for the time-sliced approach. A precalculation of the average interval is not possible. For this reason experiments have been done on a traffic system. The experiments were executed with a simulation system described in the next section.

\section{THE SIMULATION SYSTEM}

A driver has to make decisions constantly during his trip about his direction and acceleration. The selection of the direction is determined by the current position and destination of the driver. For choosing his acceleration the driver has to take the conditions on the road network and other road users into consideration. The leading vehicle has an essential influence on the acceleration.

The simulation system (Fliess 1996) used consists logically of three main components driver, vehiclefollowing, and lane model. In addition there are minor components, e.g. for describing pedestrians. The driver model reflects the behavior of the driver. He is influenced by other road users and the circumstances of the road network, like topology and lane performance. The vehicle-following model is based on the psychophysical vehicle-following model described in section two.

The circumstances of the road network are described in the lane-model. The road network can be characterized for example by the following elements:

- lanes,

- traffic lights,

- conflict points,

- sources and destinations, and

- routing tables.

A special feature of this lane model is that the length of each lane is independent of the length of the vehicles or dynamic objects. The vehicles can also be on several lanes at the same time. In many other simulation systems, there are restrictions on the length of lanes.

The essential task of the driver-model is to calculate the new acceleration of the vehicle. Correction of the acceleration is caused by the influence of the leading vehicle and the circumstances of the road network. Another special feature of this simulation model is the dynamic selection of the leading vehicle and a predictive estimation of the gap between two vehicles in conflict situations.

The simulation model was implemented in SLX (Henriksen 1996) and uses the method of the layoutbased model generation LBMG (Lorenz and Schulze 1995) for generating the essential parts of the main component lane model.

\section{EXAMPLE}

An urban road traffic system with three intersections was used for an empirical investigation. Objectives were investigation of different time-advance methods, in relation to run time and to simulation output in microscopic traffic simulation models. The investigated simulation models use the approach of psycho-physical vehicle-following modeling. Required data for describing the example were taken from real traffic projects. Figure 3 contains a schematic picture of the investigated road network. It has an extension of approximately 800 meters 
in the east-west and 250 meters in the north-south direction.

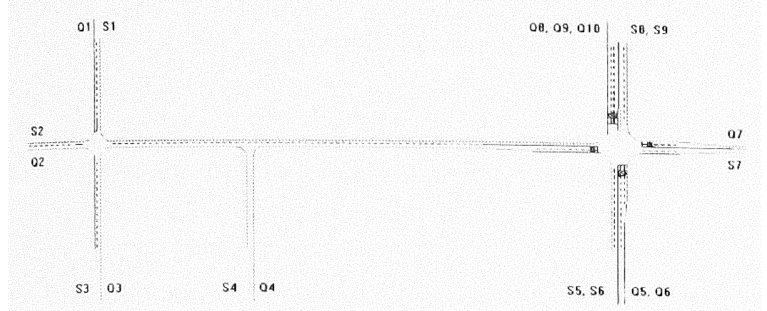

Fig. 3: Schematic Picture of the Road Network

Left intersection 1 is a simple four-way intersection. There is just one lane for each direction. Middle intersection 2 is a three-way intersection, while intersection 3 is a four-way multiple-lane intersection. Traffic lights are at intersection 1 and 3 , but not at intersection 2.

Significant parameters to describe the individual vehicles and drivers are non-deterministic. Within the given limits for these parameters an uniform distribution is used. Table 7 shows some specific vehicle parameter for the type 'car'.

Table 7: Some Specific Parameters for Vehicles

\begin{tabular}{|l|l|l|}
\hline Parameter & Low Value & Upper Value \\
\hline Car length & $3.8 \mathrm{~m}$ & $4.7 \mathrm{~m}$ \\
\hline Desired speed & $16.0 \mathrm{~m} / \mathrm{s}$ & $20.0 \mathrm{~m} / \mathrm{s}$ \\
\hline $\begin{array}{l}\text { Maximal positive } \\
\text { acceleration }\end{array}$ & $1.4 \mathrm{~m} / \mathrm{s}^{2}$ & $2.6 \mathrm{~m} / \mathrm{s}^{2}$ \\
\hline $\begin{array}{l}\text { Maximal negative } \\
\text { acceleration }\end{array}$ & $-2.5 \mathrm{~m} / \mathrm{s}^{2}$ & $-1.5 \mathrm{~m} / \mathrm{s}^{2}$ \\
\hline
\end{tabular}

\section{EMPIRICAL RESULTS}

The objective of the investigation was to determine the influence of the time-advance methods on the run time and simulation results. The simulation runs were executed on a PC with a $120 \mathrm{Mhz}$ Pentium processor. Different experiments were carried out with the example described above. The simulation model used is described in section three. The two time advance methods described, were applied to the vehicle-following and driver model components. Experiments varied the methods of time advance and the traffic load.

The values used for the experimental parameter method of time advance are:

- Event oriented, and

- Time-slice oriented with time slices of 1.0, 0.5 and $0.2 \mathrm{sec}$.
The traffic load varied among three levels. Starting with a basic load (Level 1) the load was increased by 50 percent. That means:

Level $2=1.5 *$ Level 1 and

Level $3=2.0 *$ Level 1

Each possible combination of the values of input parameters is called a scenario. The total number of scenarios is $4 * 3=12$. Twenty simulation runs with different seeds for random number generators were executed for each scenario.

\subsection{Influence on the Run Time}

Table 8 contains means and the half $90 \%$ confidential intervals of the determined run times for the simulation of one scenario (all time are in seconds).

Table 8: Mean and $90 \%$ Half Confidential Interval of Run Times

\begin{tabular}{|l|c|c|c|}
\hline & $\begin{array}{c}\text { Load } \\
\text { Level 1 }\end{array}$ & $\begin{array}{c}\text { Load } \\
\text { Level 2 }\end{array}$ & $\begin{array}{c}\text { Load Level } \\
3\end{array}$ \\
\hline Event oriented & $94 \pm 1.0$ & $150 \pm 1.7$ & $507 \pm 19.9$ \\
\hline Slice $=1$ sec. & $146 \pm 1.6$ & $245 \pm 3.8$ & $908 \pm 20.0$ \\
\hline Slice $=0.5$ sec. & $253 \pm 3.3$ & $409 \pm 6.0$ & $1342 \pm 47.6$ \\
\hline Slice $=0.2$ sec. & $584 \pm 7.3$ & $934 \pm 12.9$ & $2782 \pm 76.6$ \\
\hline
\end{tabular}

The run time grows with increasing traffic load. This trend is independent of the method of time advance. This is because the number of dynamic objects increases with increasing traffic load. The run times for the eventoriented approach are always smaller than those for the time-sliced approaches.

The results of this example support the hypothesis that the run time for the event-oriented approach in microscopic psycho-physical traffic models is smaller than those in the time-sliced approach. The unconditional requirement is the use of run-time-effective event scheduling algorithms. Figure 4 gives a graphical presentation of the average run time.

\subsection{Influence on simulation results}

The lost time for a vehicle is a typical result of simulated traffic systems. This time is the difference between the realised and the ideal travel time for a vehicle, where the ideal time would be achieved in a system with no other road users. These lost times will be calculated for every possible path from source to destination. There are 45 different paths in the example model described above. 


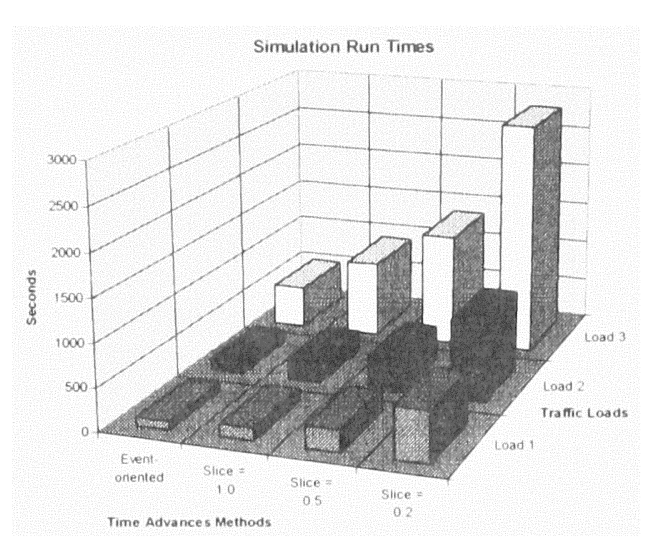

Fig. 4: Average Run Times (in sec.)

The simulation result lost time is a random variable. Twenty different simulation runs were executed to estimate the steady-state mean for this random variable. A 90 percent confidence interval was also calculated additionally. Table 9 shows the confidential interval for mean lost time on one path.

Table 9: 90\% Confidence Intervals for mean Lost Times

\begin{tabular}{|l|c|c|c|}
\hline & $\begin{array}{c}\text { Load } \\
\text { level 1 }\end{array}$ & $\begin{array}{c}\text { Load } \\
\text { level 2 }\end{array}$ & \multicolumn{1}{c|}{$\begin{array}{c}\text { Load } \\
\text { level 3 }\end{array}$} \\
\hline Event oriented & $33.0 \pm 0.9$ & $38.5 \pm 1.1$ & $79.5 \pm 11.2$ \\
\hline Slice $=0.2 \mathrm{sec}$. & $32.8 \pm 0.8$ & $38.1 \pm 1.2$ & $82.4 \pm 8.4$ \\
\hline Slice $=0.5 \mathrm{sec}$. & $33.1 \pm 0.8$ & $39.4 \pm 1.3$ & $129.9 \pm 17.1$ \\
\hline Slice $=1.0 \mathrm{sec}$. & $33.6 \pm 1.0$ & $41.5 \pm 1.4$ & $219.1 \pm 11.8$ \\
\hline
\end{tabular}

The lost time increases with growing traffic load. This trend is independent of the method of time advance. There are no significant differences in the values of lost time for traffic load level one and two. Differences occur in load level three. The values for the event-oriented approach and for the time-slice value equal 0.2 are not characterized by significant differences. The calculated confidence intervals are overlapping.

The mean for the lost time increases with increasing time slice. A growing time slice coverts a higher degree of inaccuracy of model results. Figure 5 presents 90 percent confidence intervals for lost times using traffic load level 3.

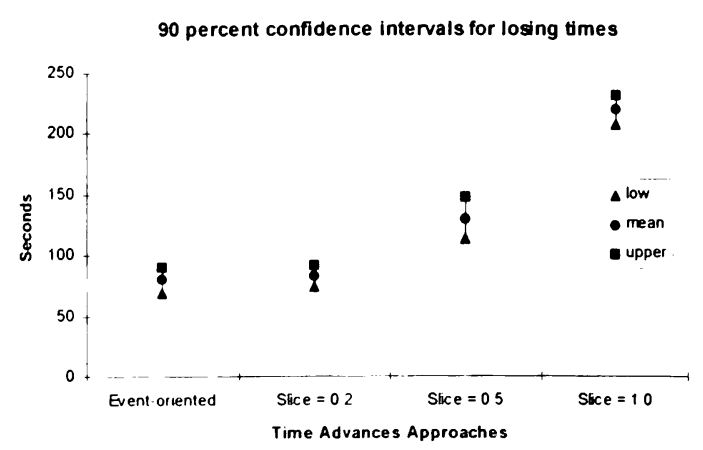

Fig. 5: 90 percent confidence intervals for lost times using traffic load level 3.

\section{CONCLUSIONS}

This paper presents results of investigations on the influence of various time-advance methods in microscopic urban traffic models. Experiments were focused on simulation run times and results. The implemented event-oriented approach, including effective algorithms for event-list handling, leads to significantly smaller run times than the time-slice approach. The use of event-oriented methods can be recommended in microscopic psycho-physical vehiclefollowing models. The kind of time-advances approach influences the accuracy of simulation results.

\section{REFERENCES}

Fliess, Th. 1996. Mikroskopische Fahrzeug-FolgeModellierung im städtischen Straßenverkehr. Master Thesis at the University of Magdeburg, Fakultät für Informatik, Germany, 1996

Heriksen, J.O. 1996. An Introduction to SLX In Proceedings of the Winter Simulation Conference 1996 (ed.) J. Charmes, D. Morrice, D. Brunner and J. Swain, pp.468-475

Hoyer, R. 1993. Modellierung und Simulation fuzzygesteuerter Lichtsignalanlagen in einem Straßennetz In 8. Symposium Simulationstechnik in Berlin 1993. (ed.) Achim Sydow, Vieweg 1993, pp 337-340

Kuehne, R. 1995. Practical Traffic Control Based on Continuum Modeling. In Proceedings of the Third International Congress on Industrial and Applied Mathematics - ICIAM 95, July 1995, Germany

Lieberman, E.B., 1991. Integrating GIS, Simulation and Animation. In Proceedings of the 1991 Winter Simulation Conference (ed.) B.L. Nelson, W.D. Kelton, and G.M. Clark, pp. 771-775

Lorenz, P. and Th. Schulze, 1995. Layout Based Model Generation. In Proceedings of the Winter Simulation 
Conference 1995 (ed.) C. Alexopoulus, K. Kang, R. Lilegdon, and D. Goldsman, pp. 728-735

McCormack, W. M. 1979. Analysis of Future Event Set Algorithm for Discrete Event Simulation, Ph.D. Dissertation, Syracuse University, 1979

Schlothauer,W., Th. Schulze, 1996. Simulation des Verkehrsablaufs an Knotenpunkten mit Grünpfeil, Project of Bundesanstalt für Straßenwesen, FP 2.9523/2, Germany

Schulze, Th. and F. Preuß 1997. Benchmarks für diskrete Simulationssysteme. In Proc. Simulation und Animation '97, Magdeburg, (ed.) O. Deussen, P. Lorenz; Society for Computer Simulation International, pp.43-55

Wiedemann, R. 1974. Simulation des Straßenverkehrsflusses In Schriftenreihe des Instituts für Verkehrswesen der Universität Karlsruhe, Germany, Heft 81974

\section{AUTHOR BIOGRAPHIES}

TOMAS SCHULZE is an Associate Professor at the Technical University in Dresden in the department for Information Systems. His research interests include modeling methodology, public systems modeling and traffic simulation. He is an active member in the ASIM, a community for simulation in Germany.

THOMAS FLIESS is a graduate student at Otto-vonGuericke University Magdeburg working towards a Ph.D. in computer science. His research area is discrete simulation in traffic models. 